\title{
Policy impacts on regulating ecosystem services: looking at the implications of 60 years of landscape change on soil erosion prevention in a Mediterranean silvo-pastoral system
}

\author{
Carlos A. Guerra . Marc J. Metzger Metzger . Joachim Maes . Teresa Pinto-Correia
}

Keywords: Regulating services _ Agricultural policy _ Landscape change _ Land cover dynamics _CAP_Montado

\begin{abstract}
:
Context Policy decisions form a major driver of land use change, with important implications for socially and environmentally susceptible regions. It is well known that there can be major unintended consequences, especially where policies are not tailored to regionally specific contexts. Objectives In this paper we assess the implications of 60 years of agricultural policies on soil erosion prevention (SEP) by vegetation, an essential regulating ecosystem service in Mediterranean Europe.

Objectives In this paper we assess the implications of 60 years of agricultural policies on soil erosion prevention (SEP) by vegetation, an essential regulating ecosystem service in Mediterranean Europe.

Methods To assess these implications we produced and analysed a time series of land cover/use and environmental conditions datasets (from 1951 to 2012) in relation to changing agricultural policies for a specific region in the southern Portugal. Set of indicators related to SEP allowed us to identify that land use intensification as increased soil erosion in the last 60 years.

Results Particularly in the last 35 years, as a consequence of head age payments for cattle, the agricultural policy had a significant effect in the density and renewal of the tree cover, resulting in drastic effects for the provision of the SEP service. These are more significant after 1986, coinciding with the implementation of several Common Agricultural Policy instruments focused on increasing the modernization and productivity capacity of farm systems.

Conclusions The results show some unintended effects of agricultural policy mechanisms on ecosystem service provision and highlight the need for context-based policies, tailored to the environmental constrains and potentials of each region.
\end{abstract}

